\title{
BMJ Open Patient-centred care for addiction treatment: a scoping review protocol
}

\author{
Kirsten Marchand, ${ }^{1,2}$ Scott Beaumont, ${ }^{1}$ Jordan Westfall, ${ }^{3}$ Scott MacDonald, ${ }^{4}$ \\ Scott Harrison, ${ }^{4}$ David C Marsh, ${ }^{5}$ Martin T Schechter, ${ }^{1,2}$ Eugenia Oviedo-Joekes ${ }^{1,2}$
}

\begin{abstract}
To cite: Marchand K, Beaumont S, Westfall $\mathrm{J}$, et al. Patient-centred care for addiction treatment: a scoping review protocol. BMJ Open 2018;8:e24588. doi:10.1136/ bmjopen-2018-024588

- Prepublication history and additional material for this paper are available online. To view these files, please visit the journal online (http://dx.doi. org/10.1136/bmjopen-2018024588).
\end{abstract}

Received 3 June 2018 Revised 27 November 2018 Accepted 28 November 2018

Check for updates

(C) Author(s) (or their employer(s)) 2018. Re-use permitted under CC BY-NC. No commercial re-use. See rights and permissions. Published by BMJ.

${ }^{1}$ School of Population and Public Health, University of British Columbia, Vancouver, British Columbia, Canada

${ }^{2}$ Centre for Health Evaluation and Outcome Sciences, Providence Health Care, St. Paul's Hospital, Vancouver, British Columbia, Canada ${ }^{3}$ Canadian Association for People Who Use Drugs, Vancouver, British Columbia, Canada ${ }^{4}$ Providence Crosstown Clinic, Providence Health Care, Vancouver, British Columbia, Canada

${ }^{5}$ Northern Ontario School of Medicine, Sudbury, Ontario, Canada

Correspondence to

Ms Kirsten Marchand;

kmarchand@cheos.ubc.ca

\begin{abstract}
Introduction Substance use disorders are chronic conditions that require a multidimensional treatment approach. Despite ongoing efforts to diversify such treatments, evidence continues to illuminate modest rates of treatment engagement and perceived barriers to treatment. Patient-centred care (PCC) is one approach that may strengthen the responsiveness of treatments for people with problematic substance use. The aim of this scoping review is to explore how the principles of PCC have been implemented and operationalised in healthcare settings for people with problematic substance use. Methods and analysis This scoping review follows the iterative stages of the Arksey and 0'Malley framework. Both empirical (from Medline, Embase, PsycINF0, CINAHL and ISI Web of Science) and grey literature references will be considered if they focused on populations with problematic substance use and described or measured PCC or one of its principles in a health-oriented context. Two reviewers will independently screen references in two successive stages of title/abstract screening and then full-text screening for references meeting title/abstract criteria. A descriptive overview, tabular and/or graphical summaries, and a directed content analysis will be carried out on extracted data. This scoping review has been registered with Open Science Framework (https://osf.io/ 5swvd/).

Ethics and dissemination This review will systematically examine the extent and nature of existing evidence of PCC in addiction research and clinical practice. Such evidence will contribute to the operationalisation of PCC for people with problematic substance use. A multidisciplinary team has been gathered to represent the needs of people with problematic substance use, healthcare providers and decision-makers. The team's knowledge users will be engaged throughout this review and will participate in dissemination activities (eg, workshops, presentations, publications, reports).
\end{abstract}

\section{INTRODUCTION}

Alcohol, tobacco and illicit substance use continues to be a significant public health concern that accounts for $11.2 \%$ of the global burden of disease and $21.1 \%$ of all deaths. People with substance use disorders are at an increased risk of mortality and morbidity ${ }^{2}$; and some may be further affected by lost family and social support, criminal justice involvement and social marginalisation. ${ }^{4}$ However,
Strengths and limitations of this study

- This is the first scoping review to systematically explore which principles of patient-centred care have been implemented and their operationalisation among people with problematic substance use.

- A multidisciplinary team composed of drug policy advocates, healthcare providers, decision-makers and academics will lead this scoping review.

- Both the population (people with problematic substance use) and concept of interest (patient-centred care) have been indexed using a variety of terms which poses a challenge to ensuring breadth of the search.

- A comprehensive search strategy has been developed in consultation with a health sciences librarian to promote a sensitive scope of empirical and grey literature sources.

- This iterative scoping review study has been registered with Open Science Framework to enhance its transparency (https://osf.io/5swvd/).

not all people with problematic substance use follow the same trajectory. Instead, there are individual variations in the personal meaning of substance use, in the intensity and frequency of use, and its associated harms. ${ }^{4-6}$ This heterogeneity in substance use disorders contributes to the complexity of its treatment.

It is increasingly accepted that there is no 'one size fits all' treatment approach for problematic substance use and that a range of treatments are required to meet the diverse needs and preferences of this population. ${ }^{5}{ }^{6}$ For example, effective pharmacological therapies are available to assist with the treatment of some, but not all, substance use disorders (eg, tobacco, alcohol, opioid dependence). ${ }^{7}$ Treatment may also include psychosocial interventions (eg, cognitive behavioural therapy, contingency management or strengths-based treatment) either in combination with pharmacological therapies or alone. ${ }^{89}$ Regardless of the treatment provided, the main goal is to engage clients in care, since treatment engagement is widely 
recognised as one of the most important predictors of substance use outcomes. ${ }^{1011}$

As such, tremendous efforts have been made towards improving treatment engagement. Examples include diversified treatment settings that offer traditional residential and hospital-based programmes, specialised outpatient programmes and, more recently, integrated service models. ${ }^{712}$ To increase the rate of detection and treatment engagement, opportunities for screening and brief intervention have also been incorporated and expanded outside of specialised substance use treatment programmes. ${ }^{13}$ Various problem-to-services matching designs have also been developed to increase successful treatment engagement by strengthening alignment between client's needs and services offered. ${ }^{14-17}$

In spite of these important developments, research continues to demonstrate quite mixed uptake of these practices, ${ }^{17}$ as well as varying rates of treatment engagement. $^{712}$ Globally, it is estimated that one out of every six people in need of substance use treatment is able to receive it; and this does not imply receipt of evidencebased or human-rights-based treatments. ${ }^{18}$ Even when examining evidence-based treatments, such as opioid agonist treatment, recent systematic reviews suggest a wide range in the rate of treatment retention (eg, from $37 \%$ to $91 \%$ at 12 -month follow-up) ${ }^{19}$ There is also a substantial body of qualitative research that has revealed several areas in which clients (and in some cases providers) have perceived challenges with engaging in treatment. A few examples include perceived provider misunderstanding of treatment goals, ${ }^{20}$ discrepancies between client and provider's treatment goals, ${ }^{21}$ a lack of treatment responsiveness to client's perceived needs, ${ }^{22} 23$ challenges with involving clients in treatment planning and delivery ${ }^{24}$ and perceived power imbalances, stigma and discrimination. ${ }^{25-27}$ This evidence suggests that there remains a need to explore how treatment processes can be designed to better respond to client's unique needs, while also considering the diversity of treatments and settings required.

Patient-centred care (PCC) is one potential approach warranting further exploration. PCC is rooted in a philosophy that 'puts the person first'. It aims to meet client's unique needs and preferences, enhance their experiences with care and involve them in all elements of treatment planning and delivery. ${ }^{28}$ Some of its origins can be traced back to Carl Rogers' client-centred therapy which emphasised unconditional positive regard, empathy and genuineness in the therapeutic process. ${ }^{29}$ Over the last two decades, as the concept of PCC has garnered increased attention across the health and social sciences, ${ }^{30}{ }^{31}$ its operationalisation has expanded beyond the role of the therapeutic relationship. For example, in nursing, empirically based conceptual frameworks ${ }^{32-34}$ agree that PCC entails an approach to care that is holistic, individualised, respectful and empowering. In medicine, the proposed frameworks converge around similar, but slightly reframed dimensions. Here, emphasis is on a biopsychosocial perspective, seeing the 'patient-asperson', enhancing the therapeutic alliance, and sharing power and responsibility. ${ }^{35-40}$ Differences in the conceptual meaning of PCC across disciplines have resulted in varying operationalisations.

That the meaning of PCC is currently somewhat discipline-specific poses challenges to determining the relationship between PCC and treatment process and outcome indicators. For example, a recent meta-analysis showed mixed effects of PCC (defined as shared control or decisions and/or consultations focused on whole person) on improved quality of care, treatment satisfaction and health outcomes. ${ }^{41}$ It also found support for generally positive effects of PCC on consultation process measures (eg, communication about treatments, levels of empathy), ${ }^{41}$ suggesting that PCC might overcome some of the challenges clients have historically experienced engaging in substance use treatment.

Indeed, elements of PCC have been recommended or defined as part of some addiction treatment approaches. ${ }^{6}$ For example, principles of respect, empathy or empowerment are integral to some treatments (eg, motivational interviewing, strengths-based treatment). However, to our knowledge, it is not known to what extent each of the dimensions of PCC have been purposefully implemented or tested across the spectrum of treatment approaches for people with problematic substance use. Bringing this evidence together in a systematic scoping review has the potential to identify cross-setting, discipline and population dimensions of PCC that have been defined, implemented and empirically explored.

\section{OBJECTIVE}

The present scoping review will systematically explore how the principles of PCC have been implemented and operationalised in healthcare settings for people with problematic substance use. Specifically, this review aims to examine the extent and nature of existing evidence of PCC in addiction research and clinical practice.

\section{METHODS AND ANALYSIS}

This scoping review methodology will apply the classic framework developed by Arksey and O'Malley, ${ }^{42}$ recent enhancements ${ }^{43-45}$ and best practices for conducting and reporting systematic reviews (ie, Preferred Reporting Items for Systematic Reviews and Meta-Analyses (PRISMA) for Protocols and Scoping Reviews (PRISMA-ScR; online supplementary 1). ${ }^{46} 47$ Accordingly, a reflexive and iterative approach will be maintained; particularly during the study screening and data extraction phases which may become more refined throughout the review. The protocol (and any potential revisions) has been registered through Open Science Framework (https://osf. io $/ 5 \mathrm{swvd} /) .{ }^{48}$ DistillerSR software for systematic reviews ${ }^{49}$ will be used by both reviewers to support screening, extraction, monitoring and the synthesis of findings. 


\section{Stage 1: Defining the research question}

The research question was developed as a broad framing of the population (ie, people with problematic substance use), the concept (ie, PCC) and the context (ie, health-oriented settings) to be explored. Thus, this scoping review asks:

1. Which PCC principles have been implemented in health-oriented settings for people with problematic substance use?

2. How have these PCC principles been operationalised when used in health-oriented settings for people with problematic substance use?

3. What outcomes from the implementation of PCC principles have been empirically measured or tested?

\section{Stage 2: Identifying relevant literature}

Our goal in developing this search strategy (online supplementary 2) is to undertake a comprehensive review of the existing evidence base. However, this particular research question poses a challenge to keyword selection due to the evolution of terms used to describe both the population and concept of interest. For instance, problematic substance use has grown from the pejorative language of the 'addict' to a health-oriented view of 'substance dependent populations' and now onto the more person-focused discourse of 'people with problematic substance use'. ${ }^{50} 51$

Likewise, as described above, conceptual frameworks of PCC have also varied, adding to the complexity of this search. To overcome this challenge, we have developed a search strategy informed by the most consistently identified and operationalised principles of PCC (in the above-mentioned frameworks), as well as keywords and $\mathrm{MeSH}$ terms from systematic reviews ${ }^{52} 53$ and empirical references ${ }^{54-56}$ previously conducted with our population of interest: (1) understanding the whole person to account for the biological, psychological and social aspects of patients' illnesses; (2) exploring the disease and illness experience to understand the personal meaning of illness and treatment for the patient; (3) finding common ground where power, knowledge and responsibility are shared between the patient and provider; and (4) enhancing the patient-provider relationship to improve the positive outcomes of treatments provided. We have also engaged in an extensive consultation process with an experienced Health Science Librarian (at the University of British Columbia) as well as the knowledge users represented in our team (authors SM and SH). The search strategy will also be peer reviewed (ie, peer review of electronic search strategies) to promote its rigour and feasibility. ${ }^{57}$

Given our interest in undertaking a comprehensive review of existing research and clinical guidelines related to PCC in the addictions field, both empirical (primary studies, previous reviews) and grey literature documents (conference abstracts, reports and clinical guidelines) will be included in our search. The search for empirical sources will be conducted in the most important electronic databases for the medical and social sciences: Medline (Ovid), Embase (Ovid), PsycINFO, CINAHL and ISI Web of Science. The search strategy has been developed in Medline (Ovid) (online supplementary 2) and will be adapted to the other databases. The search strategy will include subject headings, related terms and keywords as necessary for the research question. Boolean logic and operators (ie, 'and', 'or', 'not') will be used to combine and refine search terms and concepts.

For the grey literature search strategy, we will use recommended resources ${ }^{58}$ and consult with the Health Sciences Librarian and our team's knowledge users to devise a database specific approach. The search for abstracts, reports and clinical guidelines will be carried out in several Canadian-specific databases: British Columbia Guidelines and Protocols Databases, CPG Infobase, the Registered Nurses' Association Clinical Practice Guidelines Program and Des Libris. For international grey literature documents, we will search National Guideline Clearinghouse, TRIP, Google and Google Scholar databases.

\section{Stage 3: Study selection}

A two-stage collaborative review process will be used to select references. Eligibility criteria have been developed a priori, in consultation with the study team. The screening form will be piloted on the first 20 citations of the initial Medline (Ovid) search to test both the criteria and reviewer agreement. Two independent reviewers (authors KM and SB) will apply eligibility criteria during the initial title/abstract review. After each review stage, the reviewer's agreement will be assessed and a third reviewer (author EO-J) will be consulted in cases of disagreement, until consensus is achieved.

A title/abstract (or executive summary for reports and guidelines) will be eligible for full text screening if it:

a. Refers to people with problematic substance use (including tobacco, alcohol, cannabis, stimulant, opioid use or dual diagnoses).

b. Is about delivering PCC or one of its principles (including care that understands the whole person; explores the disease and illness experience; finds common ground and enhances the patient-provider relationship).

c. Is set in a health-oriented context (including inpatient or outpatient hospital settings, emergency departments, community-based or primary care health settings, and any specialised drug treatment or low-threshold agencies and programmes; excluding prison-based health programmes and self-help models such as narcotics or alcoholics anonymous).

d. Was published between 1 January 1960 and 1 July 2018 in English, French, Spanish, Italian, Portuguese or German.

Full text empirical articles, reports and guidelines will then be obtained for titles/abstracts meeting these above criteria and will undergo further screening. In addition to the title/abstract criteria, full texts will be included if:

e. It provided an operational definition of the PCG framework that was delivered to people with problematic substance use in the health-oriented context. 
f. It observed at least one patient outcome (eg, treatment engagement, substance use behaviours, treatment satisfaction) and/or treatment process outcome (eg, provider communication skills) of the PCC approach (this criterion pertains to empirical articles only).

As such, articles that provide only a recommendation to adopt PCC or an opinion of how PCC should be implemented in health-oriented settings for people with problematic substance use will not be included.

\section{Stage 4: Data extraction}

Results of the search will be collated in DistillerSR, ${ }^{49}$ allowing the research team to deduplicate and perform data extraction. We will follow recommended data charting methods ${ }^{42} 47$ to systematically capture relevant details for studies/reports and guidelines (table 1). Data charting forms will be piloted with the first five empirical and grey literature references and may be adapted thereafter (with input from the teams' knowledge users).

\section{Stage 5: Collating, summarising and reporting the results}

We will present a descriptive overview (including tabular and/or graphical summaries) of the eligible full texts. ${ }^{42}$ We will also summarise studies by each broader category of substances primarily used (ie, tobacco, cannabis, alcohol, opioid, stimulant, dual diagnosis). Displaying information in this way will highlight population-specific similarities and differences in PCC, its definition and

Table 1 Data extraction and charting for empirical and grey literature sources

\section{Domain/subdomain Description}

\section{General document details}

1.1 Reference type Empirical study, case study, review, commentary, report, guideline

1.2 Publication year Year of publication

1.3 Country and location Country of publication (and location if provided)

1.4 Publication language Language of publication

2. Empirical study references (if applicable)

\subsection{Research objective What was the research objective or specific question to be tested (if relevant)?}

2.2 Study design Was the study design observational, experimental or qualitative?

2.3 Study population What were the eligibility criteria? Would the population be classified as primarily: tobacco, cannabis, alcohol, opioid, stimulant or comorbid substance use and mental illness?

2.4 Patient-centred care What was the operational definition of patient-centred care used (including the definition of intervention specific principles, if available)? How long was the intervention provided or observed for?

\subsection{Context/setting \\ What health-oriented context was the PCC intervention a part of? What health professionals} were involved?

2.6 Study outcomes

For quantitative studies, what types of patient outcomes and/or process outcomes were measured (eg, treatment engagement, changes in substance use behaviours, health status, treatment satisfaction, provider communication)? For qualitative studies, what outcomes were described?

2.7 Important results What were the main results of the study? Were there any important sub-group (eg, by sex and gender, by primary substance, by healthcare provider) analyses?

2.8 Limitations

What limitations did the authors describe? What others might there be?

3. Grey literature references (if applicable)

3.1 Target audience

Is there a target audience specified for the guideline/report (eg, policy-maker/decision-maker, healthcare provider, patient/client/family)?

3.2 Reference population If available, how was the target patient population defined? Any specific eligibility criteria used? Would the population be classified as primarily: tobacco, cannabis, alcohol, opioid, stimulant or comorbid substance use and mental illness?

3.3 Patient-centred care operational definition

3.4 Context/setting

3.5 Intervention and outcomes
What was the operational definition of patient-centred care used (including the definition of specific principles, if available)?

What health-oriented context was the PCC intervention a part of? What health professionals were involved?

If applicable, was a specific patient-centred intervention described (eg, a training module, a clinical approach)? Were any patient outcomes and/or process outcomes of PCC reported (eg, treatment engagement, substance use outcomes, treatment satisfaction, provider communication)?

3.6 Programme evaluation If available, what results were reported from any ongoing programme evaluations?

PCC, patient-centred care. 
outcomes. This will facilitate the identification of future directions for research and practice. All tables and charts will include narrative summaries, relating the findings to the review's research question. Additionally, we will develop a final report of the review ${ }^{47}$ according to PRISMA-ScR guidelines. ${ }^{46}$

Given that one aim of this review is to understand how PCC has been defined and implemented in healthcare services for people with problematic substance use, a directed content analysis will be carried out on included guidelines. This approach has been deemed most suitable to the present review, since it allows existing theory to guide the coding and analysis (in our case, principles of PCC defined a priori), while still allowing new evidence to emerge. ${ }^{59}$ Specifically, we are interested in qualitatively analysing the definition of PCC adopted in the guidelines, how it was developed, which healthcare providers were involved, and any outcomes or ongoing evaluations of the programme. To do so, data from the guidelines will be imported to MAXQDA V. $12,{ }^{60}$ a qualitative analysis software program that supports a multiuser approach. This analysis will be conducted by authors KM, SB and EO-J, who have prior experience conducting thematic analysis on similar topics. ${ }^{61}{ }^{62}$ As is common in directed content qualitative analysis, ${ }^{59}$ a coding framework will be developed a priori and will then be applied by authors $\mathrm{KM}$ and SB independently. Results from this analysis will be summarised and-where relevant—numerical summaries may also be used to provide additional context to the themes (eg, number of clients treated, number of staff).

\section{Stage 6: Consultation process and engagement of knowledge users}

The ultimate aim of this review is to generate evidence that can be used to inform decision-makers and healthcare providers on the feasibility, implications and potential outcomes associated with PCC for substance use treatment. To achieve this goal, we have engaged a multidisciplinary team of knowledge users who represent the needs of: people with problematic substance use, healthcare providers and decision-makers. Consulting with the teams' healthcare providers and decision-makers (authors SH, SM and DCM) will promote a methodology that reflects the realities of patient-provider roles and the healthcare system's organisation. Also, our team's drug policy knowledge user (author JW) represents a national organisation of people who use drugs and this critical perspective will ensure that all aspects of this review are rooted in the client-centred needs of this diverse population. The specific contributions of the knowledge users to each stage of this review have been defined throughout. At this time, knowledge users have reviewed early drafts of the search strategy, identifying additional terms that are important for inclusion given the population, concept and contexts of interest (eg, trauma-informed care and culturally-safe care). Knowledge users have also provided several grey-literature references (clinical guidelines and reports) to be considered for inclusion. As the project continues to evolve, all knowledge users will be involved in supporting the interpretation of findings and their dissemination.

\section{Patient and public involvement}

This scoping review protocol has engaged the expertise of a national organisation of people who use(d) drugs through the involvement of this organisations' president. This knowledge user (author JW) has made contributions to the development of the research question and will also be extensively involved during the interpretation and dissemination phases of this project.

\section{ETHICS AND DISSEMINATION}

As substance use disorders are increasingly recognised as a chronic condition often marked by cycles of relapse and recovery, the public healthcare system is considering how existing treatment and intervention approaches can be optimised to meet the long-term and evolving goals of clients. ${ }^{18}$ Adopting patient-centred or person-centred approaches may increase the responsiveness of existing treatments to individual client needs, expectations and preferences. To our knowledge, this review will be the first to systematically examine the extent and nature of existing evidence of PCC in addiction research and clinical practice.

Our dissemination strategy will use traditional approaches, including open-access peer-reviewed publication(s), scientific presentations and a report. Additionally, we are committed to promoting further action based on the potential findings of this review. Therefore, we will host a half-day round-table meeting-bringing together people with problematic substance use, healthcare providers (from diverse settings) and decision-makers to brainstorm potential opportunities for future areas of research and clinical practice work. For example, we may engage in a concept mapping exercise, using the findings of this review to integrate stakeholders' knowledge, interpretations and priorities into practice. The multidisciplinary nature of this team will facilitate and support our goal of bringing together these different representatives together.

Acknowledgements We would also like to acknowledge Ursula Ellis, Health Sciences Librarian (University of British Columbia), who has provided invaluable expertise to the development and refinement of the search strategy of this scoping review.

Contributors KM (review guarantor) led the design and conceptualisation of this review and drafted the protocol with primary support from SB, MTS and E0-J. SB, SH and EO-J were involved in refining the search strategy, including keywords. SM, SH, DCM and JW were involved in establishing eligibility criteria and data extraction forms. All authors provided feedback on the manuscript and approval to the publishing of this protocol manuscript.

Funding Funding for this scoping review has been provided by the Canadian Institutes of Health Research (Operating Grant: Opioid Crisis Knowledge Synthesis OCK-156780).

Competing interests None declared.

Patient consent Not required. 
Provenance and peer review Not commissioned; externally peer reviewed.

Open access This is an open access article distributed in accordance with the Creative Commons Attribution Non Commercial (CC BY-NC 4.0) license, which permits others to distribute, remix, adapt, build upon this work non-commercially, and license their derivative works on different terms, provided the original work is properly cited, appropriate credit is given, any changes made indicated, and the use is non-commercial. See: http://creativecommons.org/licenses/by-nc/4.0/.

\section{REFERENCES}

1. Gakidou E, Afshin A, Abajobir AA, et al. Global, regional, and national comparative risk assessment of 84 behavioural, environmental and occupational, and metabolic risks or clusters of risks, 1990-2016: a systematic analysis for the Global Burden of Disease Study 2016. The Lancet 2017;390:1345-422.

2. Holford TR, Meza R, Warner KE, et al. Tobacco control and the reduction in smoking-related premature deaths in the United States, 1964-2012. JAMA 2014;311:164-71.

3. Whiteford HA, Degenhardt L, Rehm J, et al. Global burden of disease attributable to mental and substance use disorders: findings from the Global Burden of Disease Study 2010. Lancet 2013;382:1575-86.

4. Nutt DJ, King LA, Phillips LD. Independent Scientific Committee on Drugs. Drug harms in the UK: a multicriteria decision analysis. Lancet 2010;376:1558-65.

5. Hammer G. Trust and relationship as cornerstones of successful psychotherapy. In: Tatarsky A, Tatarsky A, eds. Harm reduction psychotherapy: a new treatment for drug and alcohol problems. Lanham, MD, US: Jason Aronson, 2002:106-35.

6. Abou-Saleh MT, Tarter RE, Salloum IM, et al. Substance use disorders. In: Mezzich JE, Botbol M, Christodoulou GN, eds. Person centered psychiatry. Cham, Switzerland: Springer International Publishing, 2016:325-43.

7. Substance Abuse and Mental Health Services Administration. Chapter 4, Early Intervention, Treatment, And Management of Substance Use Disorders. In: Facing addiction in America: the surgeon general's report on alcohol, drugs, and health. Washington (DC): Office of the Surgeon General (US), 2016.

8. Jhanjee S. Evidence based psychosocial interventions in substance use. Indian J Psychol Med 2014;36:112-8.

9. Magill M, Ray LA. Cognitive-behavioral treatment with adult alcohol and illicit drug users: a meta-analysis of randomized controlled trials. J Stud Alcohol Drugs 2009;70:516-27.

10. Hubbard RL, Craddock SG, Flynn PM, et al. Overview of 1-year follow-up outcomes in the Drug Abuse Treatment Outcome Study (DATOS). Psychology of Addictive Behaviors 1997;11:261-78.

11. Simpson DD, Joe GW, Rowan-Szal GA. Drug abuse treatment retention and process effects on follow-up outcomes. Drug Alcohol Depend 1997;47:227-35.

12. Substance Abuse and Mental Health Services Administration. Chapter 6, Health Care Systems and Substance Use Disorders. In: Facing addiction in America: the surgeon general's report on alcohol, drugs, and health. Washington (DC): US Department of Health and Human Services, 2016.

13. Babor TF, McRee BG, Kassebaum PA, et al. Screening, brief intervention, and referral to treatment (SBIRT). Subst Abus 2007;28:7-30.

14. Marsh JC, Cao D, Shin HC. Closing the need-service gap: gender differences in matching services to client needs in comprehensive substance abuse treatment. Soc Work Res 2009;33:183-92.

15. Hser YI, Polinsky ML, Maglione M, et al. Matching clients' needs with drug treatment services. J Subst Abuse Treat 1999;16:299-305.

16. Mattson ME, Babor T, Cooney N, et al. Matching patients with alcohol disorders to treatments: clinical implications from project MATCH. Journal of Mental Health 1998;7:589-602.

17. Alexander JA, Nahra TA, Lemak $\mathrm{CH}$, et al. Tailored treatment in the outpatient substance abuse treatment sector: 1995-2005. J Subst Abuse Treat 2008;34:282-92.

18. United Nations Office on Drugs and Crime. International standards for the treatment of drug use disorders. Geneva: World Health Organization, 2017.

19. Timko C, Schultz NR, Cucciare MA, et al. Retention in medicationassisted treatment for opiate dependence: a systematic review. $J$ Addict Dis 2016;35:22-35.

20. Rance J, Treloar C. "We are people too": consumer participation and the potential transformation of therapeutic relations within drug treatment. Int J Drug Policy 2015;26:30-6.
21. Joosten EAG, De Weert-Van Oene GH, Sensky T, et al. Treatment goals in addiction healthcare: the perspectives of patients and clinicians. Int J Soc Psychiatry 2011;57:263-76.

22. McNeil R, Kerr T, Pauly B, et al. Advancing patient-centered care for structurally vulnerable drug-using populations: a qualitative study of the perspectives of people who use drugs regarding the potential integration of harm reduction interventions into hospitals. Addiction 2016;111:685-94.

23. Pauly BB, McCall J, Browne AJ, et al. Toward cultural safety: nurse and patient perceptions of illicit substance use in a hospitalized setting. ANS Adv Nurs Sci 2015;38:121-35.

24. Bryant J, Saxton M, Madden A, et al. Consumers' and providers' perspectives about consumer participation in drug treatment services: is there support to do more? What are the obstacles? Drug Alcohol Rev 2008;27:138-44.

25. Fischer J, Neale J. Involving drug users in treatment decisions: an exploration of potential problems. Drugs: Education, Prevention \& Policy 2008;15:161-75.

26. Strike C, Rufo C. Embarrassing, degrading, or beneficial: patient and staff perspectives on urine drug testing in methadone maintenance treatment. J Subst Use 2010;15:303-12.

27. Palis H, Marchand K, Peng D, et al. Factors associated with perceived abuse in the health care system among long-term opioid users: a cross-sectional study. Subst Use Misuse 2016;51:763-76.

28. Berwick DM. What 'patient-centered' should mean: confessions of an extremist. Health Aff 2009;28:w555-65.

29. Rogers C. On becoming a person. a therapist's view of psychotherapy. Boston: Houghton Mifflin Company, 1961.

30. Institute of Medicine (IOM). Crossing the quality chasm: a new health system for the 21st century. 2001.

31. Beaulieu MD. President's message: toward a patient-centred health care system. Can Fam Physician 2013;59:109,10.

32. Slater L. Person-centredness: a concept analysis. Contemp Nurse 2006;23:135-44.

33. Hobbs JL. A dimensional analysis of patient-centered care. Nurs Res 2009;58:52-62.

34. Morgan S, Yoder LH. A concept analysis of person-centered care. J Holist Nurs 2012;30:6-15.

35. Mead N, Bower P. Patient-centredness: a conceptual framework and review of the empirical literature. Soc Sci Med 2000;51:1087-110.

36. Stewart M. Towards a global definition of patient centred care. BMJ 2001;322:444-5.

37. Robinson JH, Callister LC, Berry JA, et al. Patient-centered care and adherence: definitions and applications to improve outcomes. J Am Acad Nurse Pract 2008;20:600-7.

38. McMillan SS, Kendall E, Sav A, et al. Patient-centered approaches to health care: a systematic review of randomized controlled trials. Med Care Res Rev 2013;70:567-96.

39. Scholl I, Zill JM, Härter M, et al. An integrative model of patientcenteredness - a systematic review and concept analysis. PLoS One 2014;9:e107828.

40. Santana MJ, Manalili K, Jolley RJ, et al. How to practice person-centred care: a conceptual framework. Health Expect 2018;21:429-40.

41. Dwamena F, Holmes-Rovner M, Gaulden CM, et al. Interventions for providers to promote a patient-centred approach in clinical consultations. Cochrane Database Syst Rev 2012;12:Cd003267.

42. Arksey $\mathrm{H}, \mathrm{O}^{\prime}$ Malley L. Scoping studies: towards a methodological framework. Int J Soc Res Methodol 2005;8:19-32.

43. Levac D, Colquhoun H, O'Brien KK. Scoping studies: advancing the methodology. Implement Sci 2010;5:69.

44. Daudt HM, van Mossel C, Scott SJ. Enhancing the scoping study methodology: a large, inter-professional team's experience with Arksey and O' Malley's framework. BMC Med Res Methodol 2013;13:48

45. Colquhoun HL, Levac D, O'Brien KK, et al. Scoping reviews: time for clarity in definition, methods, and reporting. J Clin Epidemiol 2014:67:1291-4.

46. Tricco AC, Lillie E, Zarin W, et al. PRISMA extension for scoping reviews (PRISMA-ScR): checklist and explanation. Ann Intern Med 2018;169:467.

47. Joanna Briggs Institute. The Joanna Briggs institute reviewers'manual 2015: methodology for JBI scoping review. Australia: Joanna Briggs Institute, 2015.

48. Center for Open Science. Open science framework. Charlottesville, VA, 2011.

49. Evidence Partners. DistillerSR: systematic review and literature review software [program]. Ottawa, Canada: Evidence Partners, 2016. 
50. Kelly JF, Saitz R, Wakeman S, et al. Language, substance use disorders, and policy: the need to reach consensus on an "addictionary". Alcoholism Treatment Quarterly 2016;34:116-23.

51. Broyles LM, Binswanger IA, Jenkins JA, et al. Confronting inadvertent stigma and pejorative language in addiction scholarship: a recognition and response. Subst Abus 2014;35:217-21.

52. Barrio P, Gual A. Patient-centered care interventions for the management of alcohol use disorders: a systematic review of randomized controlled trials. Patient Prefer Adherence 2016;10:1823-45.

53. Friedrichs $\mathrm{A}$, Spies $\mathrm{M}$, Härter M, et al. Patient preferences and shared decision making in the treatment of substance use disorders: a systematic review of the literature. PLoS One 2016;11:e0145817.

54. Schwartz RP, Kelly SM, Mitchell SG, et al. Patient-centered methadone treatment: a randomized clinical trial. Addiction 2017;112:454-64.

55. Joosten E, de Weert G, Sensky T, et al. Effect of shared decisionmaking on therapeutic alliance in addiction health care. Patient Prefer Adherence 2008;2:277-85.
56. Joosten EA, De Weert-Van Oene GH, Sensky T, et al. Treatment goals in addiction healthcare: the perspectives of patients and clinicians. Int J Soc Psychiatry 2011;57:263-76.

57. McGowan J, Sampson M, Salzwedel DM, et al. PRESS Peer review of electronic search strategies: 2015 guideline statement. J Clin Epidemiol 2016;75:40-6.

58. Canadian Agency for Drugs and Technologies in Health. Grey matters: a practical tool for searching health-related grey literature. 2015 https://www.cadth.ca/resources/finding-evidence/grey-matters (Accessed 22 Nov 2017).

59. Hsieh HF, Shannon SE. Three approaches to qualitative content analysis. Qual Health Res 2005;15:1277-88.

60. MAXQDA. Qualitative Analysis Software [program]. 2015.

61. Oviedo-Joekes E, Marchand K, Palis H, et al. Predictors of treatment allocation guesses in a randomized controlled trial testing doubleblind injectable hydromorphone and diacetylmorphine for severe opioid use disorder. Addict Res Theory 2017;25:263-72.

62. Oviedo-Joekes E, Marchand K, Lock K, et al. A chance to stop and breathe: participants' experiences in the North American Opiate Medication Initiative clinical trial. Addict Sci Clin Pract 2014;9:21-31. 